\title{
A study of computer-based learning model for students with dyslexia
}

\begin{abstract}
Dyslexia is one of the most common Specific Learning Difficulties (SpLDs) in the world. Students with dyslexia have poor fluency in reading, writing, spelling, speech, short-term memory, and also other related disorders. In addition emotion is recognised as important as the cognitive difficulty that affects dyslexia learning. Students with dyslexia often suffer emotions like frustration and low self-esteem due to lack of achievement. As a result, they may develop behaviour difficulty or perceive by others as misbehaviours. In order to address the dyslexia learning difficulties several computer-based learning models have been introduced. The computer-based learning model found to be interesting, user-friendly, attractive and supportive. In this paper, we present a study of computer-based learning model to support dyslexia students. The work is crucial to provide a basis for developing a computer-based learning model that addresses dyslexia language-based learning difficulties that considers both student's cognitive and emotion. In addition, the study also explores the uses of machine learning (ML) approach to improve effectiveness of the learning process.
\end{abstract}

Keyword: Behaviour; Cognitive; Dyslexia; Learning model; Machine learning 